\title{
Spectral-Domain Optical Coherence Tomography Characteristics of Intermediate Age-Related Macular Degeneration
}

\author{
Jessica N. Leuschen, MD1, Stefanie G. Schuman, MD ${ }^{1}$, Katrina P. Winter, BS ${ }^{1}$, Michelle N. \\ McCall, MCAPM, BA ${ }^{1}$, Wai T. Wong, MD, PhD ${ }^{2}$, Emily Y. Chew, MD ${ }^{2}$, Thomas Hwang, MD $^{3}$, \\ Sunil Srivastava, MD ${ }^{4}$, Neeru Sarin, MBBS ${ }^{1}$, Traci Clemons, PhD $^{5}$, Molly Harrington, MBA ${ }^{5}$, \\ and Cynthia A. Toth, MD ${ }^{1}$
}

${ }^{1}$ Duke Eye Center, Durham, North Carolina ${ }^{2}$ National Eye Institute, Bethesda, Maryland ${ }^{3}$ Devers Eye Institute, Portland, Oregon ${ }^{4}$ Emory Eye Center, Atlanta, Georgia ${ }^{5}$ EMMES Corporation, Rockville, Maryland

\section{Abstract \\ Purpose-Describe qualitative spectral-domain optical coherence tomography (SD-OCT) characteristics of eyes classified as intermediate age-related macular degeneration (nonadvanced AMD) from Age-Related Eye Disease Study 2 (AREDS2) color fundus photography (CFP) grading.}

Design-Prospective cross-sectional study.

Participants-We included 345 AREDS2 participants from 4 study centers and 122 control participants who lack CFP features of intermediate AMD.

Methods-Both eyes were imaged with SD-OCT and CFP. The SD-OCT macular volume scans were graded for the presence of 5 retinal, 5 subretinal, and 4 drusen characteristics. In all, 314 AREDS2 participants with $\geq 1$ category-3 AMD eye and all controls each had 1 eye entered into SD-OCT analysis, with 63 eyes regraded to test reproducibility.

Main Outcome Measures-We assessed SD-OCT characteristics at baseline.

Results-In 98\% of AMD eyes, SD-OCT grading of all characteristics was successful, detecting drusen in $99.7 \%$, retinal pigment epithelium (RPE) atrophy/absence in $22.9 \%$, subfoveal geographic atrophy in $2.5 \%$, and fluid in or under the retina in 25.5\%. Twenty-eight percent of AMD eyes had characteristics of possible advanced AMD on SD-OCT. Two percent of control eyes had drusen on SD-OCT. Vision loss was not correlated with foveal drusen alone, but with foveal drusen that were associated with other foveal pathology and with overlying focal hyperreflectivity. Focal hyperreflectivity over drusen, drusen cores, and hyper- or hyporeflectivity of drusen were also associated with RPE atrophy.

(C) 2012 by the American Academy of Ophthalmology.

Correspondence: Cynthia A. Toth, MD, Duke Eye Center, Box 3802, Erwin Road, Durham, NC 27710.

Financial Disclosure(s):

The authors have made the following disclosures:

Cynthia A. Toth: Research support-Genentech, Sirion Therapeutics, Bioptigen, Inc, Alcon Laboratories, Inc; potential royaltiesBioptigen, Inc, Alcon Laboratories, Inc.

Duke University has an equity and intellectual property interest in Bioptigen.

The remaining authors do not have any financial disclosures. 
Conclusions-Macular pathologies in AMD can be qualitatively and reproducibly evaluated with SD-OCT, identifying pathologic features that are associated with vision loss, RPE atrophy, and even possibly the presence of advanced AMD not apparent on CFP. Qualitative and detailed SD-OCT analysis can contribute to the anatomic characterization of AMD in clinical studies of vision loss and disease progression.

Age-related macular degeneration (AMD), a major retinal disease with genetic and environmental factors, is the most common cause of legal blindness in industrialized nations. ${ }^{1-5} \mathrm{~A}$ wide spectrum of phenotypic morphologies, disease characteristics, functional consequences, and rates of progression have been described for AMD. ${ }^{1-6}$ Drusen are the hallmark lesions of early and intermediate AMD, and the presence of large, confluent drusen and focal hyperpigmentation as identified on color fundus photography (CFP) constitute risk factors for progression to advanced AMD in the forms of choroidal neovascularization $(\mathrm{CNV})$ and central geographic atrophy (CGA). ${ }^{6-10}$ Anatomic lesions of AMD that relate to the risk of disease advancement may be useful in identifying at-risk patients and guiding preventative measures that reduce vision loss from AMD. ${ }^{11}$

Traditionally, CFP is the imaging modality that has been used in the clinical staging of AMD for epidemiologic studies and the definition of AMD-associated lesions. ${ }^{12,13}$ Although CFP provides an en face view of the retina, optical coherence tomography (OCT) provides a cross-sectional visualization that allows for the characterization of microstructural alterations in the different laminae of the retina. ${ }^{14-16}$ Spectral-domain (SD) OCT technology currently provides increased imaging resolution and acquisition speed compared with previous time-domain OCT imaging instruments. ${ }^{17-22}$

Previous studies have shown OCT to be clinically useful in identifying AMD pathology as well as studying disease progression. Schuman et $\mathrm{al}^{23}$ used SD-OCT to examine the early degeneration of the neurosensory retina in AMD, which revealed photoreceptor loss and thinning of the outer nuclear layer above drusen, changes that cannot be visualized using CFP. ${ }^{23}$ Brar et $\mathrm{al}^{24}$ found that imaging features on high-resolution OCT correlated well with those seen with fundus autofluorescence imaging and postulated that OCT may be a better predictor of progression to GA than fundus autofluorescence. Bearelly et $\mathrm{al}^{25}$ reproducibly measured photoreceptor loss at GA margins with OCT and suggested that OCT may be useful in following the progression of geographic atrophy.

Although the anatomic features of AMD in the various stages of the disease as visualized using CFP have been previously described and characterized in a number of major clinical trials, ${ }^{10,26}$ a similar qualitative characterization of anatomic features of AMD as visualized by SD-OCT has not been performed in a large AMD population. Khanifar et $\mathrm{al}^{27} \mathrm{had}$ previously established a simple grading system for evaluating drusen ultrastructure on SDOCT which was applied to a single retinal scan in each eye. The present study extended this grading system to SD-OCT scan sets covering the entire macula. Our goal here was to detect and characterize the full spectrum of retinal pathologies and drusen characteristics that are visible on SD-OCT imaging of nonadvanced AMD. We have in this report provided qualitative descriptions of the lesion phenotypes found and quantitative measures for the frequency at which they occur. These SD-OCT characteristics may relate to different stages of AMD severity within the range of nonadvanced AMD, and may also relate to the risk of progression to advanced AMD, and therefore constitute potential markers for risk stratification. Although the current report performs an initial characterization of SD-OCT findings from a cross-sectional evaluation, longitudinal analyses of future data arising from AREDS2 will help to ascertain how these characteristics can help to stage AMD severity and predict AMD progression. 


\section{Methods}

The institutional review boards of the following 4 AREDS2 clinics approved this study: Devers Eye Institute, Duke Eye Center, Emory Eye Center, and the National Eye Institute. Informed consent was obtained from all participants, adhering to the tenets of the Declaration of Helsinki. The AREDS2 Ancillary SD-OCT (A2A SD-OCT) Study was registered at www.clinicaltrials.gov (ClinicalTrials.gov Identifier: NCT00794498) on August 13, 2008. Of the 364 eligible AREDS2 participants from these sites, 345 (95\%) consented to participate in the A2A SD-OCT Study. Inclusion criteria for the AREDS2 included age between 50 and 85 years, bilateral large drusen ( $\geq 125$ microns) or large drusen in 1 eye and advanced AMD in the fellow eye, and CFP assessed by the Fundus Photograph Reading Center to be of adequate quality. The study eyes have to lack signs of advanced AMD but may contain geographic atrophy that did not include foveal center, consistent with the AREDS definition of category 3 AMD. ${ }^{28}$ Patients with ocular diseases other than AMD in either eye or a history of retinal surgery were excluded. Inclusion criteria for control participants were (1) age between 50 and 85 years, (2) adequate CFP quality, (3) absence of ocular diseases in either eye or a history of retinal surgery, and (4) absence of AMD, large drusen (>125 microns) in the macula of either eye, any geographic atrophy or CNV as determined by clinical examination and on CFP. Best-corrected visual acuity data (determined by Early Treatment Diabetic Retinopathy Study letters) was obtained to evaluate the impact of age on visual acuity and to relate anatomic structure to function among the 313 category 3 AMD study eyes and the 122 control eyes (1 eye from each subject).

For AREDS2 participants, AMD category was assigned by clinical examination and CFP images according to the AREDS Report No. 1 grading scale by the Fundus Photograph Reading Center. We considered AMD categories 1, 2, and 3 nonadvanced stages of AMD; AMD category 4 included CNV and CGA, types of advanced AMD. ${ }^{28}$ The breakdown of the AMD categorization of both eyes of 345 participants based on CFP grader assessment is found in Figure 1.

Of 345 AMD participants imaged during the study period from December 19, 2006 to November 30, 2009, adequate SD-OCT scans were obtained in both eyes of 342 AMD participants. In $3 \mathrm{AMD}$ participants, $\geq 1$ eye was not imaged adequately owing to insufficient media clarity. These eyes were excluded from analysis. In 28 AMD participants, both eyes were assigned to category 4 AMD from CFP grading and were also excluded from analysis. In cases of bilateral category $3 \mathrm{AMD}$, the right eye was designated the study eye so that only a single eye from each participant would be included in the analyses. Participants with only 1 eye assigned to category 3 AMD $(n=130)$ contributed their category 3 AMD eye to the analysis. This created a set of 314 study eyes with category 3 AMD. A group of 122 control participants who met age eligibility for AREDS 2 were imaged in both eyes from December 18,2008 , to February 28, 2011. One eye each from 3 participants was excluded owing to an ocular abnormality in that eye. For the remaining 119 participants, the right eye was designated as the study eye for the first half of the participants and the left eye designated as the study eye for the latter half of subjects, which resulted in only 1 eye from each participant included in the analysis.

Certified imagers from each Study Site performed SD-OCT imaging using the Bioptigen Tabletop SD-OCT system (Research Triangle Park, NC). Two linear and 4 volumetric scans were captured in each eye following a standard imaging protocol. These included two $6.7 \times$ 6.7-mm volumetric scans captured at $0^{\circ}$ and $90^{\circ}(1000 \mathrm{~A}$ scans per B scan with 67 -micron spacing between the $100 \mathrm{~B}$ scans per volume), and two $3.35 \times 3.35-\mathrm{mm}$ volumetric scans (500 A scans per B scan with 17 -micron spacing between each of the $200 \mathrm{~B}$ scans) at $0^{\circ}$ and 
$90^{\circ}$. Scan quality was assessed by a certified grader and was assigned a designation of good, fair, or poor. Reasons for decreased scan quality were also recorded.

Three scan sets covering the entire macula (including two $6.7 \times 6.7-\mathrm{mm}$ volumetric scans at $0^{\circ}$ and $90^{\circ}$ and one $3.35 \times 3.35-\mathrm{mm}$ volumetric scan either at $0^{\circ}$ or $90^{\circ}$, based on best scan quality) were analyzed per eye by certified SD-OCT graders masked to participant clinical and photographic data. Each scan was graded based on presence or absence of 5 retinal, 5 subretinal, and 4 drusen characteristics that are defined in Table 1 (available at http:// aaojournal.org), and depicted in Figures 2 and 3. The definition of drusen on SD-OCT was based on work by Khanifar et $\mathrm{al}^{27}$ and Jain et al, ${ }^{29}$ in which SD-OCT characteristics of drusen were described, and in Jain et al ${ }^{29}$ were compared pixel-by-pixel to color fundus photographic overlay. Each of the 3 volume scans were graded for the presence or absence of pathologic characteristics. If $\geq 1$ of 3 volume scans had a positive grade for a characteristic, the characteristic was judged to have a final grade of "present." If all 3 volume scans were negative for a characteristic, then the characteristic was judged to have a final grade of "absent." We also separately compared the difference between the grade from a single volume and the final grade. Additionally, graders noted whether each of the morphologic abnormalities was located within a $0.67-\mathrm{mm}$ diameter of the foveal center. The grader identified the foveal center on the retinal image projected from the volume combined with appearance of the foveal depression on cross-sectional scans. Additional analyses were performed in these eyes to discover associations between the grades of retinal morphology and the grades of drusen.

A secondary analysis was performed for eyes with SD-OCT pathologic features of intra- and subretinal fluid that may suggest the presence of possible CNV, which would be inconsistent with the CFP classification of category 3 AMD. An experienced retina clinician and certified SD-OCT grader (SGS) reviewed the scans for these eyes and scored the pathologies in 2 categories: (1) suspicious for neovascular AMD or (2) fluid may be attributed to another cause, such as isolated small intraretinal cystoid structure or fluid associated with vitreomacular traction or epiretinal membrane (ERM).

To assess the reproducibility of SD-OCT grading for AMD eyes, 2 independent graders graded a randomly selected subset of 63 study eyes from AREDS2 participants at different times. The median time interval between the 2 gradings was 17.5 months (range, 5-27).

To determine the effect of foveal retinal pathologies on visual acuity, subgroups of category 3 AMD study eyes containing foveal retinal pathology (defined as pathology on SD-OCT images within 333 microns of the foveal center) were compared with category 3 AMD study eyes without any foveal pathology. Age group was included as a covariate in the model because visual acuity was significantly different across age groups. However, the interaction between foveal group and age group was not significant and was not included as a covariate.

In the initial analysis, foveal pathologies were not considered exclusively because, other than drusen, no other foveal pathology was present alone in $>2$ eyes in the AMD group. A secondary analysis of visual acuity by foveal pathology alone was performed for drusen.

All analyses were performed using SAS version 9.2 (SAS Institute Inc, Cary, NC). The prevalence of each retinal pathology and drusen characteristic among subgroups of study eyes was compared using the Pearson's chi-square test or the Fisher exact test (for small sample sizes). To assess the agreement between 2 independent graders on the presence or absence of a retinal lesion, percent agreement and Fleiss' kappa were calculated.

Relative risk was computed to assess whether eyes with certain drusen characteristics were more or less likely to also have other drusen characteristics. This was assessed using the 
GENMOD procedure with a binomial probability distribution. The autoregressive structure of the working correlation matrix was used.

The effect of age on visual acuity was calculated using a generalized linear model (SAS Version 9.2); all pair-wise comparisons and adjusted $P$ values for multiple comparisons were calculated using Tukey's post hoc analysis. The generalized linear model procedure was also used to conduct a 2-way analysis of variance to test differences in visual acuity among eyes with and without foveal retinal pathologies.

\section{Results}

The 314 participants with category 3 AMD selected for this study included 297 (94.59\%) Caucasians and $17(5.41 \%)$ non-Caucasians; there were 129 (41.08\%) male and 185 $(58.92 \%)$ female participants. The average age of category 3 AMD participant was $74 \pm 7.5$ years. The 122 control participants enrolled this study consisted of $118(96.72 \%)$ Caucasians and $4(3.28 \%)$ non-Caucasians; there were $52(42.62 \%)$ male and $70(57.38 \%)$ female participants. The control participants met age eligibility for AREDS2 ( 255 but $<85$ ); however, their mean age was $67 \pm 7.3$ years, which was 7.4 years younger than the AMD group $(P<0.0001)$.

Of the 941 scan volumes reviewed for the 314 eyes in this baseline analysis, scan quality was "good" in 76\% ( $\mathrm{n}=717)$, "fair" in 19\% $(\mathrm{n}=178)$, and "poor" in $5 \%(\mathrm{n}=43)$, and the quality scores were similar between scan volumes $\left(6.7 \times 6.7-\mathrm{mm}\right.$ volumetric scans at $0^{\circ}, 6.7$ $\times 6.7-\mathrm{mm}$ volumetric scans at $90^{\circ}$, and $3.35 \times 3.35-\mathrm{mm}$ volumetric scans either at $0^{\circ}$ or $\left.90^{\circ}\right)$. One eye had 2 scan volumes captured instead of 3 . Reasons for poor scan quality (ordered from most to least common) were participant motion or lack of fixation (22\%); poor signal owing to OCT system, focus, or opacity in visual axis (21\%); participant blink artifact $(15 \%)$; lack of foveal centration (when the foveal center is not reasonably central in the volume, the area sampled on SD-OCT may not cover the macula; $12 \%$ ); scan extending off anterior or posterior margin of scan window (7\%); and severe pathology that obscured border between retinal and subretinal structures $(5 \%)$. The SD-OCT scans of poor quality could generally still be graded. Only 6 of 314 study eyes (2\%) had $\geq 1$ SD-OCT characteristics that could not be scored. Of these, only 1 eye could not be scored for any SDOCT characteristics. All of the control eyes except 1 could be scored for all SD-OCT characteristics. The graders were unable to read vitreomacular attachment (VMA) in 1 control eye owing to poor scan quality.

The final SD-OCT grade was based on review of 3 volume scans. In comparing the grade from a single SD-OCT volume with the final SD-OCT grade for each eye, there were several instances where a single volume grade did not identify pathology that was found on either of the other 2 volumes. Ordered from most to least common, these missed pathologies were intraretinal cysts (7.64\%), VMA (5.41\%), ERM (3.5\%), subretinal fluid (3.18\%), retinal pigment epithelium (RPE) atrophy or absence, other subretinal lesion (1.59\%), and drusen $(0.32 \%)$.

Based on the kappa statistic, good agreement was found between independent graders for the majority of SD-OCT findings and there was excellent agreement between graders for drusen in the fovea and RPE atrophy or absence, whereas drusen with high reflectivity had the lowest percent agreement between graders and a very low kappa statistic (Table 2; available at http://aaojournal.org). When considering these agreements, it is important to recognize, however, that several characteristics were present or absent in almost all eyes in the study, which is reflected in the prevalence index (Table 2, available at http:// aaojournal.org). In addition, agreement between independent graders was $100 \%$ for drusen; 
therefore, a kappa statistic could not be calculated (Table 2, available at http:// aaojournal.org).

The primary analysis consisted of the prevalence of SD-OCT characteristics among 313 category 3 AMD study eyes from 314 participants (Fig 4), with a comparison to those in 122 control eyes. The 5 retinal and 5 subretinal pathologies along with 4 drusen characteristics are defined in Table 1 (available at http://aaojournal.org), with examples in Figures 2 and 3. The prevalence of vitreoretinal interface pathology in the forms of partial vitreous separation with VMA or ERM was comparable between the AMD eyes in 20.6\% VMA and 38.2\% ERM and control eyes in $22.1 \%$ VMA and $43.4 \%$ ERM. The SD-OCT characteristics suggestive of fluid within or beneath the retina, such as intraretinal cysts, subretinal fluid, or subretinal pre-RPE lesion, were found in $25.5 \%$ of study AMD eyes, but were either rarely found or completely absent in control eyes. Atrophy or absence of the RPE, found in $22.9 \%$ of AMD study eyes, was absent in control eyes. In 2.5\% of AMD study eyes, en face SDOCT projections revealed a patch of RPE atrophy exceeding a diameter of 360 microns that also extended beneath the center of the fovea. This is consistent with AREDS grading of advanced AMD owing to subfoveal geographic atrophy if it had been identified on color fundus photographic grading. Although drusen with medium internal reflectivity were nearly ubiquitous in AMD study eyes (98.7\%), drusen with low and high reflectivity were less prevalent ( $43.3 \%$ and $60.5 \%$, respectively). In the retina overlying drusen, focal hyperreflectivity was much less common than photoreceptor thinning (Fig 4) in AMD study eyes. Drusen were identified on SD-OCT images in 19 control eyes (15.6\%) and in 3 eyes (2.5\%) these were within the central $0.67 \mathrm{~mm}$.

The relationships between certain drusen characteristics of 313 AMD eyes were analyzed. Internal reflectivity of drusen was associated with several other drusen characteristics. Eyes with either low- or high-reflective drusen were more likely to have drusen with an internal core than eyes lacking these drusen features (relative risk of having a core among eyes with low-reflective drusen compared with eyes without low-reflective drusen: 3.07 [95\% confidence interval [CI], 2.04-4.63]; relative risk of having a core among eyes with highreflective drusen compared with eyes without high-reflective drusen: 1.85 [95\% CI, 1.202.84]). A significant association was found between the presence of drusen with internal cores and the presence of focal high reflectivity above drusen; cores were more likely to be prevalent in eyes with focal high reflectivity above drusen than in eyes without focal high reflectivity (relative risk, 3.99; 95\% CI, 2.35-6.78). The reverse was also true; among eyes containing drusen with a core, focal high reflectivity above drusen was significantly more likely to be prevalent than in eyes containing drusen without a core (RR, $1.83 ; 95 \% \mathrm{CI}$, $1.55-2.18)$.

Certain drusen characteristics were significantly associated with RPE atrophy or absence. Among study eyes with RPE atrophy or absence $(\mathrm{n}=72$ eyes), low-reflective drusen $(P<0.0001)$, high-reflective drusen $(P=0.0388)$, drusen with cores $(P<0.0001)$, and focal high reflectivity above drusen $(P<0.0001)$ were significantly more prevalent than in eyes without RPE atrophy or absence as described in Table 3 (available at http://aaojournal.org).

We reviewed the SD-OCT characteristics for guidance in defining the category of AMD in 313 study eyes. A secondary review was performed on the 86 eyes (27.4\%) with category 3 AMD according to CFP that on SD-OCT grading contained intraretinal cysts, subretinal fluid, serous PED, or pathology suspicious for CNV (nondrusen subretinal lesion). Of these, 41 (13.1\%) eyes had either a single intraretinal cyst (Fig 2C) or other retinal pathologies that were judged to have etiologies other than neovascular AMD, such as vitreomacular traction. The remaining 45 eyes (14.3\%) were judged to be suspicious for advanced neovascular AMD, which might warrant further clinical investigation (Fig 2D, E). Fourteen of these eyes 
had intraretinal cysts alone, 4 had subretinal fluid alone, 8 had subretinal pathology alone, and 4 had PED alone; 13 had 2 of these pathologies; 4 had 3 of these pathologies; and 2 eyes contained all 4 of these pathologies. Additionally, 8 of 313 eyes (2.5\%) with category 3 AMD were found to have RPE atrophy involving the foveal center whose greatest lateral dimension exceeded 360 microns (mean lateral dimension, 866 microns) suggesting the presence of category 4 disease in the form of CGA (Fig 2G). Because fluorescein angiography was not obtained in the AREDS2 study protocol at the time of SD-OCT imaging, no correlative angiography data were available.

The overall mean ETDRS value for category 3 AMD study eyes was $80.4 \pm 8.96$ letters (median, 82; Snellen equivalent, 20/25) and for controls was 84 \pm 5.46 (median, 85; Snellen equivalent, 20/20). Subgroups based on age were created to delineate the relationship between visual acuity and age (Fig 5). Mean visual acuity decreased significantly with increasing age in the AMD group $(P<0.0001)$, but not in the control group $(P=0.3139)$. Mean visual acuity among AMD participants 50 to 59 years of age was significantly higher than among participants aged 70 to $79(P=0.0006)$ and 80 to $89(P<0.0001)$. Additionally, mean visual acuity in AMD age group 60 to 69 was significantly higher than in AMD age groups 70 to $79(P=0.0002)$ and 80 to 89 years $(P<0.0001)$.

In AMD eyes, mean visual acuity score (adjusted for age) decreased with all foveal pathologies compared with eyes with no foveal pathologies (Fig 6). This decrease was significant in all subgroups except eyes with foveal VMA. Visual acuity was lowest among subgroups containing foveal subretinal fluid and foveal RPE atrophy or absence, although we did not test for significance in acuity between the pathologies. Of all of the foveal pathologies evaluated, eyes with foveal drusen had the smallest decrease in visual acuity compared with eyes without foveal drusen ( -4.7 letters), although the mean visual acuity score was still significantly decreased compared with study eyes without foveal drusen $(P=$ 0.009). It should be noted that visual acuity may be influenced by a number of foveal pathologies in combination, and the specific foveal pathology in each subgroup was not determined to be exclusively responsible for the decrease in visual acuity score. Except for drusen, the foveal pathologies did not occur alone in the foveal center in $>2$ eyes. When compared with mean acuity for eyes with no pathology $(87.3 \pm 2.3)$, there was no difference in acuity for the 189 eyes with drusen alone $(83.8 \pm 0.5$ letters; $P=0.057)$, whereas the 93 eyes with drusen with other pathology had a lower mean acuity (78.6 \pm 1.3 letters; $P=$ 0.0002). In addition, although the 162 eyes with foveal drusen and hyperreflective foci had significantly lower visual acuity ( $81.4 \pm 0.8$ letters) than the 121 eyes without the hyperreflective foci ( $83.2 \pm 0.8$ letters; $P=0.048$, age adjusted), when looking at eyes with foveal drusen alone (189 eyes; e.g., no geographic atrophy) there was no difference in acuity between the 96 eyes with (83.6 \pm 0.7 letters) and the 93 eyes without hyperreflective foci $(84.0 \pm 0.7 ; P=0.625)$. The 271 eyes with foveal drusen and photoreceptor layer (PRL) thinning did not show a significant difference in visual acuity compared with the 11 eyes without thinning $(P=0.08)$. In contrast with the AMD group, the 122 control eyes had mean visual acuity of $84.0 \pm 5.46$, had fewer pathologies near the foveal center ( 3 eyes with VMA, 10 with ERM, and VMA in 2 of these eyes; CME in 1 eye and drusen in 3 eyes), with no impact on visual acuity.

\section{Discussion}

This is the first large-population study qualitatively characterizing retinal pathologies and drusen characteristics seen on SD-OCT in eyes with nonadvanced AMD, reporting their prevalence in AMD eyes and aged non-AMD eyes and correlating the presence of the pathology with acuity. A wide spectrum of drusen morphologies was observed on SD-OCT, 
including all the drusen types previously described by Khanifar et $\mathrm{al}^{27}$ and Fleckenstein et al. ${ }^{30}$

Unlike previous studies in which a single foveal scan was analyzed, $\geq 100$ scans across the macula were reviewed for each study eye in this study. This enabled a comprehensive survey of pathologic features in the macula, and allowed their prevalence and associations with each other to be ascertained. This study also demonstrated the broad applicability of SD-OCT in the description and definition of AMD pathology in a clinical study setting; graders of SD-OCT images were able to definitively score the presence or absence of all intra- and subretinal characteristics in $>98 \%$ of eyes with category 3 AMD, and achieve good to excellent intergrader agreement for many of the scored characteristics.

The morphology selected for analysis from SD-OCT imaging as seen in Table 1 (available at http://aaojournal.org) was guided by findings in preliminary studies of nonadvanced AMD. ${ }^{23,27,30}$ Because SD-OCT images are analyzed from cross-sectional images, many of the features considered in SD-OCT grading, such as VMA, intraretinal cystoid structures, PRL thickness, subretinal fluid, drusen cores, or hyperreflective foci over drusen, may not be comparable with conventional CFP grading. Jain et al, ${ }^{29}$ in a pixel-by-pixel comparison of areas of the fundus designated as drusen on CFP and those designated as drusen on SDOCT (using the same drusen definition as this study), found $82 \%$ agreement between these modalities. Although most disagreement occurred at the margins of drusen, they also identified disagreements such as when pigment on color photographs masked the underlying druse, which was visible on SD-OCT. ${ }^{29}$ Many of the CFP findings, particularly several that relate to risk of disease progression, are based, however, on en face quantitative measurements or estimates of area, for example, largest drusen size, drusen area, and number of zones with predominant soft drusen. ${ }^{10}$ These are not readily performed with manual SD-OCT grading. Jain et $\mathrm{al}^{29}$ reported a trend toward greater drusen detection on SD-OCT when drusen size increased or hyperpigmentation was present and a trend toward greater detection of the smaller drusen by CFP. Several groups have demonstrated methods for quantitative measurement of drusen from SD-OCT imaging through computer-assisted segmentation. ${ }^{31-33}$ Quantitative measurements from these study eyes will be addressed in future reports.

Drusen were nearly ubiquitous in eyes categorized with category 3 AMD by CFP and the majority of eyes contained drusen of medium reflectivity. Drusen characteristics on SDOCT may correlate with AMD progression risk and provide further insight into the pathophysiology of AMD. There was a significant association between drusen with internal cores and focal high reflectivity above drusen, and both of these were more prevalent in eyes containing drusen of low or high internal reflectivity. Additionally, focal high reflectivity over drusen, drusen with low and high internal reflectivity, and drusen with cores were more prevalent in eyes with RPE atrophy or absence. Just as drusen type on CFP has been shown to be crucial in estimating an individual's risk of AMD, drusen morphology on SD-OCT may prove to be important factor in risk stratification. ${ }^{34}$ This clustering suggests that atypical reflectivity of drusen and focal hyperreflectivity over drusen may be indicators of processes associated with RPE atrophy. The utility of these drusen characteristics as predictive factors will be tested in the longitudinal A2A SD-OCT Study.

Our interest in hyperreflectivity over drusen related to the early findings of Schuman et al, ${ }^{23}$ who observed hyperreflective foci overlying drusen in $41 \%$ of AMD eyes (assessing a single scan through the foveal center), and postulated that these are a precursor to larger pigment clumps visible on CFP and may represent progression of disease, and of Khanifar et al, ${ }^{27}$ who correlated highly reflective intraretinal foci on SD-OCT with hyperpigmentation on CFP, which has already been shown to be a risk factor for progression to advanced 
AMD. 1,35,36 The association of hyperreflectivity over drusen with abnormal internal drusen reflectivity may be revealing of pathologic processes such as inflammatory changes occurring within drusen ${ }^{37}$ and in the outer retina ${ }^{38}$ that may drive the progression of AMD. The cellular basis underlying outer retinal hyperreflectivity is currently unknown but may represent the infiltration of inflammatory cells (e.g., retinal microglia) that can induce disruptions in the RPE layer, ${ }^{39}$ leading to pigmentary changes visible on CFP. Longitudinal SD-OCT imaging of nonadvanced AMD may have the potential to reveal pathogenic cellular interactions that are revealing of mechanisms of AMD progression. In addition, future studies that correlate AMD risk genotypes with the prevalence of SD-OCT AMD pathologies can also be used to explore the use of SD-OCT features as potential OCT imaging biomarkers.

Internal reflectivity of drusen may be useful for determining progression to advanced disease; however, high drusen reflectivity had lower percent agreement between graders (Table 2, available at http://aaojournal.org), pointing to the difficulty in grading this drusen characteristic. This may be owing to determination of the percent of the drusen, which seems hyperreflective compared with mid-reflective or to the presence of very small drusen with mid to high reflectivity. We are evaluating additional standardization processes for this manual grading, versus automated grading of internal reflectivity of drusen to better address this. Further studies will allow us to determine whether specific internal drusen reflectivity characteristics lead to advanced disease.

Not only did SD-OCT confirm the CFP assessment of AMD, but SD-OCT also provided additional information that was not obtained from CFP. Subfoveal RPE atrophy or absence was observed in $2.5 \%$ of eyes identified as category 3 AMD by CFP. If foveal RPE atrophy or absence on SD-OCT coincides with CGA, then CFP was less sensitive than SD-OCT in detecting this pathology. Additionally, 27.4\% of eyes contained $\geq 1$ SD-OCT characteristics suggestive of CNV and/or intra- or subretinal fluid. Review of the SD-OCT scans by a clinician identified at least half of these (45 eyes) with signs of fluid suspicious for advanced AMD. All of these eyes were originally graded nonadvanced based on CFP.

The presence of SD-OCT characteristics suggestive of advanced disease in CFP-graded category 3 AMD eyes may indicate that either AMD severity was underestimated by CFP, or these SD-OCT pathologies are not always associated with advanced disease. In eyes with neovascular AMD, Stopa et al ${ }^{17}$ found that SD-OCT could identify the presence and extent of pathologic features (such as $\mathrm{CNV}$, macular edema, and subretinal fluid) that were not identified with conventional imaging, including CFP, fundus autofluorescence, and the fluorescein angiogram. Although the location and extent of AMD pathologies were not assessed in this qualitative study, it is possible that review of SD-OCT images detected signs of advanced AMD that were missed by CFP and on clinical examination. However, on secondary review, half of the SD-OCT findings of fluid did not necessarily seem to be suspicious for an AMD process; for example, these were associated with vitreoretinal interface or without notable subretinal or sub-RPE pathology. Without fluorescein angiography, we cannot relate the SD-OCT findings in this study to classic methods used to define presence or absence of CNV. Intra- or subretinal fluid may be unassociated with a $\mathrm{CNV}$ process and yet could be a marker for future pathway of disease progression. These A2A patients will be followed over 4 years to determine the role of these SD-OCT characteristics in risk stratification.

In a cross-sectional, population-based study of visual acuity in AMD, Klein et $\mathrm{al}^{40}$ found, while controlling for other factors (age, central cataract, and glaucoma), that each of the early age-related maculopathy lesions (soft indistinct drusen, RPE degeneration, and increased retinal pigment, as seen on color fundus photographs) was associated with a 
decrease in visual acuity of approximately $\mathcal{s}$ letters when compared with eyes without these lesions. Klein et $\mathrm{al}^{41}$ also found that age was a significant and independent predictor of poor visual acuity, and this was also found in the AMD eyes in this study (Fig 5). After adjusting for age, the presence of foveal pathology on SD-OCT significantly correlated with a lower mean visual acuity. This was true of all foveal pathologies except VMA. The most dramatic decrease in visual acuity was seen among eyes containing foveal subretinal fluid and foveal RPE atrophy or absence. Foveal drusen were the largest subcategory and were also associated with a significant decrease in acuity; however, secondary analysis revealed that a decrease in acuity was associated with drusen in the presence of another foveal pathology (ERM, VMA, cystoid edema, subretinal fluid, subretinal lesion, RPE atrophy) and not owing to drusen alone. In eyes with foveal drusen without excluding other foveal pathology, those with hyperreflective foci over drusen had significantly poorer vision than those without, and this poor acuity may be owing to the association between hyperreflective foci and RPE atrophy. It is notable that eyes with RPE atrophy where RPE atrophy was within $333 \mu \mathrm{m}$ of the fovea were generally those with the lowest acuity (Table 4; available at http:// aaojournal.org). In contrast, in eyes with foveal drusen alone, there was no difference in acuity whether hyperreflective foci were present or absent. In addition, eyes with photoreceptor thinning over drusen did not have decreased acuity compared with the few eyes without this thinning. Although the small number of eyes without PRL thinning limits the power of this analysis, this measure may prove to be a poor anatomic correlate with acuity. Foveal drusen alone were not associated with significant decrease in acuity. In more recent studies, large drusen have been associated with loss of photoreceptor outer segments ${ }^{23}$ and with focal decrease in retinal sensitivity. ${ }^{42,43}$ Future analysis of the photoreceptor inner segment band or external limiting membrane in SD-OCT images may provide better correlation with function than PRL thinning. In control eyes, there were too few eyes with drusen or foveal pathology for meaningful analysis (Table 5; available at http://aaojournal.org).

By characterizing SD-OCT pathologies in a baseline set of category 3 AMD eyes and following pathologic changes over time, we hope to identify specific SD-OCT characteristics that precede disease progression. To this end, Yehoshua et $\mathrm{a}^{44}$ used quantitative assessment of SD-OCT to document drusen volume changes over time and reported their interest in using change in drusen volume as a clinical trial endpoint. We are especially interested in drusen morphologic patterns and their relevance to disease progression. Great variability of drusen microstructure was observed on SD-OCT and may provide unique insight into the active pathophysiology of pathways of disease. We are examining the correlation of drusen characteristics with fellow eye disease and with subsequent progression of disease in this study. By following these SD-OCT characteristics longitudinally, a simpler system of grading may emerge as we learn which characteristics are most predictive of advanced disease - perhaps an SD-OCT corollary to the AREDS simplified severity scale. ${ }^{45}$

This study demonstrates that qualitative SD-OCT features of macular pathologies in AMD (1) can be reproducibly graded in a research setting, (2) share associations with each other and with vision loss, and (3) may provide greater detail than CFP in discerning anatomic features of AMD and in detecting advanced AMD. In future studies, we will directly correlate SD-OCT characteristics with CFP pathologies, which may prove useful in establishing an SD-OCT grading scale for AMD. In addition, quantitative analysis of SDOCT pathology provides valuable information in terms of AMD severity. ${ }^{44}$ We are currently developing an automated segmentation algorithm to quantify drusen volume on SD-OCT. ${ }^{31}$ These measurements will provide more information for risk stratification of patients with category $3 \mathrm{AMD}$, which will be useful for intervention to prevent vision loss. 


\section{Acknowledgments}

This project (ClinicalTrials.gov identifier: NCT00734487, August 13, 2008) was funded in part by Genentech grant (IST-4400S), Bioptigen (equipment), and Alcon Laboratories (unrestricted startup grant).

\section{References}

1. Klein R, Klein BE, Knudtson MD, et al. Fifteen-year cumulative incidence of age-related macular degeneration: the Beaver Dam Eye Study. Ophthalmology. 2007; 114:253-62. [PubMed: 17270675]

2. Tomany SC, Wang JJ, Van Leeuwen R, et al. Risk factors for incident age-related macular degeneration: pooled findings from 3 continents. Ophthalmology. 2004; 111:1280-7. [PubMed: 15234127]

3. Eye Diseases Prevalence Research Group. Prevalence of age-related macular degeneration in the United States. Arch Ophthalmol. 2004; 122:564-72. [PubMed: 15078675]

4. Wang JJ, Rochtchina E, Lee AJ, et al. Ten-year incidence and progression of age-related maculopathy: the Blue Mountains Eye Study. Ophthalmology. 2007; 114:92-8. [PubMed: 17198852]

5. Augood CA, Vingerling JR, de Jong PT, et al. Prevalence of age-related maculopathy in older Europeans: the European Eye Study (EUREYE). Arch Ophthalmol. 2006; 124:529-35. [PubMed: 16606879]

6. Sunness JS, Rubin GS, Applegate CA, et al. Visual function abnormalities and prognosis in eyes with age-related geographic atrophy of the macula and good visual acuity. Ophthalmology. 1997; 104:1677-91. [PubMed: 9331210]

7. Sunness JS, Margalit E, Srikumaran D, et al. The long-term natural history of geographic atrophy from age-related macular degeneration: enlargement of atrophy and implications for interventional clinical trials. Ophthalmology. 2007; 114:271-7. [PubMed: 17270676]

8. Wang JJ, Foran S, Smith W, Mitchell P. Risk of age-related macular degeneration in eyes with macular drusen or hyperpigmentation: the Blue Mountains Eye Study cohort. Arch Ophthalmol. 2003; 121:658-63. [PubMed: 12742843]

9. Holz FG, Bindewald-Wittich A, Fleckenstein M, et al. FAM-Study Group. Progression of geographic atrophy and impact of fundus autofluorescence patterns in age-related macular degeneration. Am J Ophthalmol. 2007; 143:463-72. [PubMed: 17239336]

10. Age-Related Eye Disease Study Research Group. The Age-Related Eye Disease Study severity scale for age-related macular degeneration: AREDS report no. 17. Arch Ophthalmol. 2005; 123:1484-98. [PubMed: 16286610]

11. Loewenstein, A. The significance of early detection of age-related macular degeneration: Richard \& Hinda Rosenthal Foundation lecture. Retina; the Macula Society 29th annual meeting; 2007. p. 873-8.

12. Klein R, Davis MD, Magli YL, et al. The Wisconsin Age-Related Maculopathy Grading System. Ophthalmology. 1991; 98:1128-34. [PubMed: 1843453]

13. Age-Related Eye Disease Study Research Group. The Age-Related Eye Disease Study system for classifying age-related macular degeneration from stereoscopic color fundus photographs: the Age-Related Eye Disease Study report number 6. Am J Ophthalmol. 2001; 132:668-81. [PubMed: 11704028]

14. Huang D, Swanson EA, Lin CP, et al. Optical coherence tomography. Science. 1991; 254:117881. [PubMed: 1957169]

15. Hee MR, Baumal CR, Puliafito CA, et al. Optical coherence tomography of age-related macular degeneration and choroidal neovascularization. Ophthalmology. 1996; 103:1260-70. [PubMed: 8764797]

16. Toth CA, Narayan DG, Boppart SA, et al. A comparison of retinal morphology viewed by optical coherence tomography and by light microscopy. Arch Ophthalmol. 1997; 115:1425-8. [PubMed: 9366674] 
17. Stopa M, Bower BA, Davies E, et al. Correlation of pathologic features in spectral domain optical coherence tomography with conventional retinal studies. Retina. 2008; 28:298-308. [PubMed: 18301035]

18. Pieroni CG, Witkin AJ, Ko TH, et al. Ultrahigh resolution optical coherence tomography in nonexudative age related macular degeneration. Br J Ophthalmol. 2006; 90:191-7. [PubMed: 16424532]

19. Srinivasan VJ, Wojtkowski M, Witkin AJ, et al. High-definition and 3-dimensional imaging of macular pathologies with high-speed ultrahigh-resolution optical coherence tomography. Ophthalmology. 2006; 113:2054-65. [PubMed: 17074565]

20. Schmidt-Erfurth U, Leitgeb RA, Michels S, et al. Three-dimensional ultrahigh-resolution optical coherence tomography of macular diseases. Invest Ophthalmol Vis Sci. 2005; 46:3393-402. [PubMed: 16123444]

21. Drexler W, Sattmann H, Hermann B, et al. Enhanced visualization of macular pathology with the use of ultrahigh-resolution optical coherence tomography. Arch Ophthalmol. 2003; 121:695-706. [PubMed: 12742848]

22. de Boer JF, Cense B, Park BH, et al. Improved signal-to-noise ratio in spectral-domain compared with time-domain optical coherence tomography. Opt Let. 2003; 28:2067-9. [PubMed: 14587817]

23. Schuman SG, Koreishi AF, Farsiu S, et al. Photoreceptor layer thinning over drusen in eyes with age-related macular degeneration imaged in vivo with spectral-domain optical coherence tomography. Ophthalmology. 2009; 116:488-96. [PubMed: 19167082]

24. Brar M, Kozak I, Cheng L, et al. Correlation between spectral-domain optical coherence tomography and fundus autofluorescence at the margins of geographic atrophy. Am J Ophthalmol. 2009; 148:439-44. [PubMed: 19541290]

25. Bearelly S, Chau FY, Koreishi A, et al. Spectral domain optical coherence tomography imaging of geographic atrophy margins. Ophthalmology. 2009; 116:1762-9. [PubMed: 19643488]

26. Maguire MG, Alexander J, Fine SL. Complications of Age-related Macular Degeneration Prevention Trial (CAPT) Research Group. Characteristics of choroidal neovascularization in the Complications of Age-related Macular Degeneration Prevention Trial. Ophthalmology. 2008; 115:1468-73. [PubMed: 18486222]

27. Khanifar AA, Koreishi AF, Izatt JA, Toth CA. Drusen ultrastructure imaging with spectral domain optical coherence tomography in age-related macular degeneration. Ophthalmology. 2008; 115:1883-90. [PubMed: 18722666]

28. Age-Related Eye Disease Study Research Group. The Age-Related Eye Disease Study (AREDS): design implications. AREDS report no 1 Control Clin Trials. 1999; 20:573-600.

29. Jain N, Farsiu S, Khanifar AA, et al. Quantitative comparison of drusen segmented on SD-OCT versus drusen delineated on color fundus photographs. Invest Ophthalmol Vis Sci. 2010; 51:487583. [PubMed: 20393117]

30. Fleckenstein M, Charbel Issa P, Helb HM, et al. High-resolution spectral domain-OCT imaging in geographic atrophy associated with age-related macular degeneration. Invest Ophthalmol Vis Sci. 2008; 49:4137-44. [PubMed: 18487363]

31. Chiu SJ, Izatt JA, O'Connell RV, et al. Validated automatic segmentation of AMD pathology including drusen and geographic atrophy in SD-OCT images. Invest Ophthalmol Vis Sci. 2012; 53:53-61. [PubMed: 22039246]

32. Gregori G, Wang F, Rosenfeld PJ, et al. Spectral domain optical coherence tomography imaging of drusen in nonexudative age-related macular degeneration. Ophthalmology. 2011; 118:1373-9. [PubMed: 21388687]

33. Baumann B, Gotzinger E, Pircher M, et al. Segmentation and quantification of retinal lesions in age-related macular degeneration using polarization-sensitive optical coherence tomography. $\mathrm{J}$ Biomed Opt. 2010; 15:061704. [PubMed: 21198152]

34. Williams MA, Craig D, Passmore P, Silvestri G. Retinal drusen: harbingers of age, safe havens for trouble. Age Ageing. 2009; 38:648-54. [PubMed: 19726434]

35. Buch H, Vinding T, la Cour M, et al. Risk factors for age-related maculopathy in a 14-year followup study: the Copenhagen City Eye Study. Acta Ophthalmol Scand. 2005; 83:409-18. [PubMed: 16029262] 
36. van Leeuwen R, Klaver CC, Vingerling JR, et al. The risk and natural course of age-related maculopathy: follow-up at 6 1/2 years in the Rotterdam study. Arch Ophthalmol. 2003; 121:51926. [PubMed: 12695249]

37. Hageman GS, Luthert PJ, Victor Chong NH, et al. An integrated hypothesis that considers drusen as biomarkers of immune-mediated processes at the RPE-Bruch's membrane interface in aging and age-related macular degeneration. Prog Retin Eye Res. 2001; 20:705-32. [PubMed: 11587915]

38. Gupta N, Brown KE, Milam AH. Activated microglia in human retinitis pigmentosa, late-onset retinal degeneration, and age-related macular degeneration. Exp Eye Res. 2003; 76:463-71. [PubMed: 12634111]

39. Ma W, Zhao L, Fontainhas AM, et al. Microglia in the mouse retina alter the structure and function of retinal pigmented epithelial cells: a potential cellular interaction relevant to AMD. PLoS One. 2009; 4:e7945. serial online, Available at: http://www.plosone.org/article/info\%3Adoi \%2F10.1371\%2Fjournal.pone.0007945. [PubMed: 19936204]

40. Klein R, Wang Q, Klein BE, et al. The relationship of age-related maculopathy, cataract, and glaucoma to visual acuity. Invest Ophthalmol Vis Sci. 1995; 36:182-91. [PubMed: 7822146]

41. Klein R, Klein BE, Linton KL, De Mets DL. The Beaver Dam Eye Study: visual acuity. Ophthalmology. 1991; 98:1310-5. [PubMed: 1923372]

42. Iwama D, Tsujikawa A, Ojima Y, et al. Relationship between retinal sensitivity and morphologic changes in eyes with confluent soft drusen. Clin Experiment Ophthalmol. 2010; 38:483-8. [PubMed: 20584018]

43. Midena E, Vujosevic S, Convento E, et al. Microperimetry and fundus autofluorescence in patients with early age-related macular degeneration. Br J Ophthalmol. 2007; 91:1499-503. [PubMed: 17504849]

44. Yehoshua Z, Wang F, Rosenfeld PJ, et al. Natural history of drusen morphology in age-related macular degeneration using spectral domain optical coherence tomography. Ophthalmology. 2011; 118:2434-41. [PubMed: 21724264]

45. Age-Related Eye Disease Study Research Group. A simplified severity scale for age-related macular degeneration: AREDS report no. 18. Arch Ophthalmol. 2005; 123:1570-4. [PubMed: 16286620] 


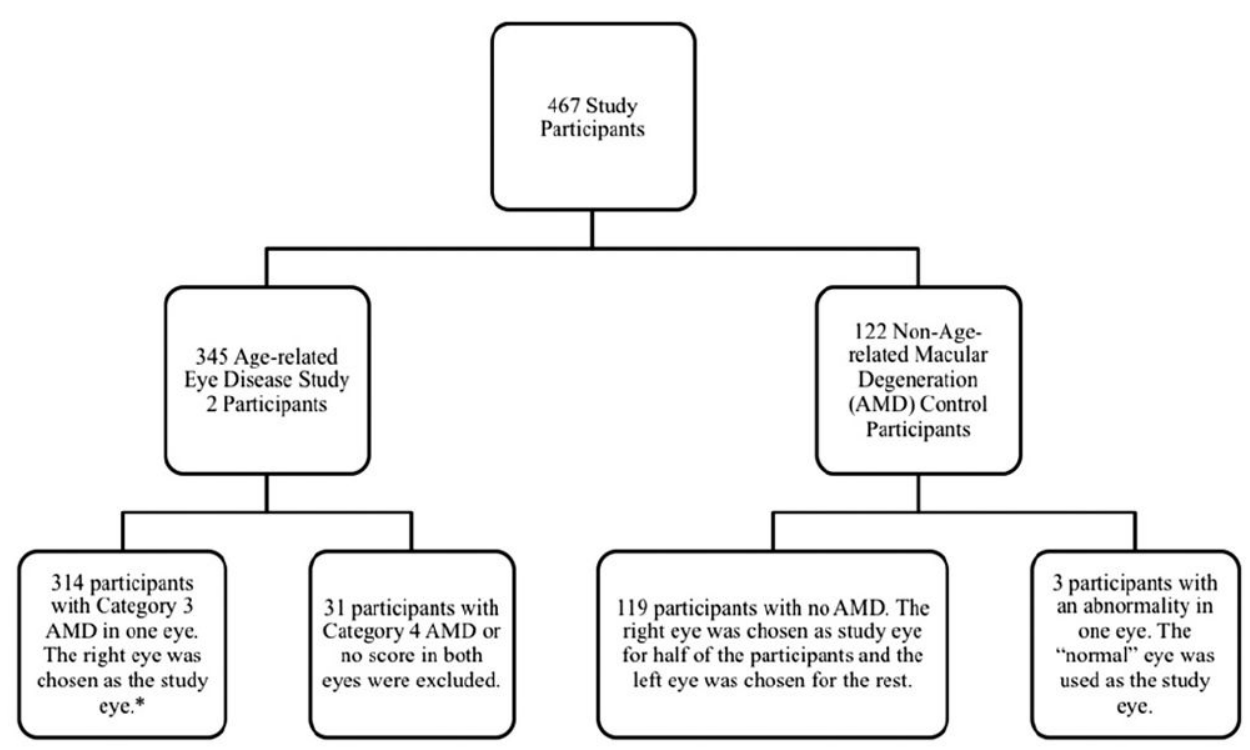

Figure 1.

Study eye designation and age-related macular degeneration (AMD) categorization of both eyes from 345 participants by color fundus photography grading. *One of the 314 eyes could not be graded. 

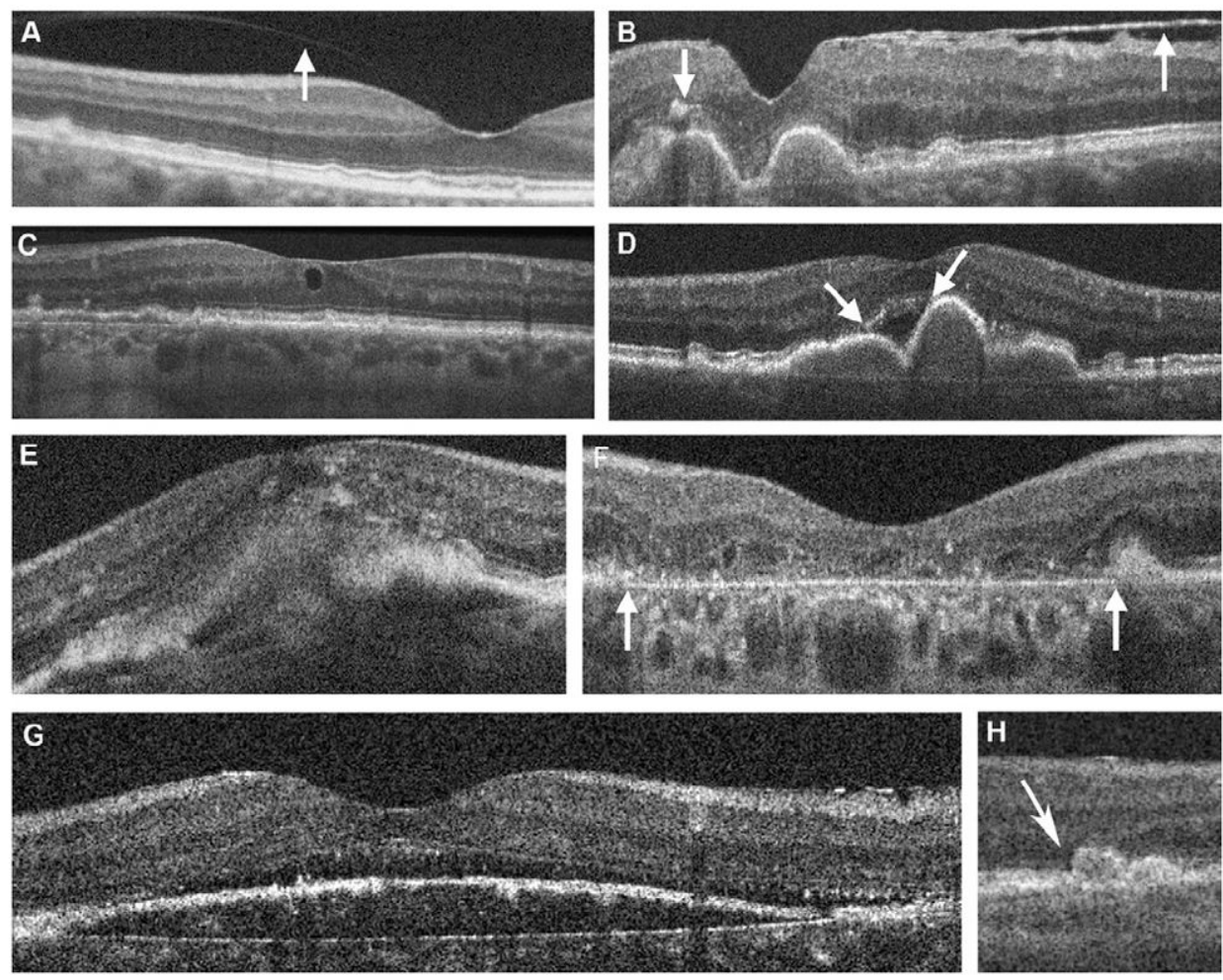

Figure 2.

Examples of spectral-domain optical coherence tomography morphology in study eyes with age-related macular degeneration (AMD). All of these eyes had been graded as category 3 AMD (nonadvanced) on color fundus photographs. The spectral-domain optical coherence tomography morphology terms are defined in Table 1 (available at http://aaojournal.org). To reduce the noise in linear B-scan images, varying numbers of B-scans were summed using Image J software. A, Vitreomacular attachment (arrow). B, Epiretinal membrane (upward arrow) and focal high reflectivity over druse (downward arrow) within the outer retina and distinct from normal variation in retinal reflectivity. C, Intraretinal cystoid structure. D, Subretinal fluid over large contiguous drusen (arrows). E, Subretinal pathology that likely represents choroidal neovascularization with overlying intraretinal fluid (cystoid edema). F, A broad area of retinal pigment epithelial atrophy (geographic atrophy) extends through the fovea with overlying photoreceptor loss and increase in reflectivity of the underlying choroid. G, Subretinal fluid at the foveal center. H, Photoreceptor layer thinning over drusen (left druse, with arrow). 

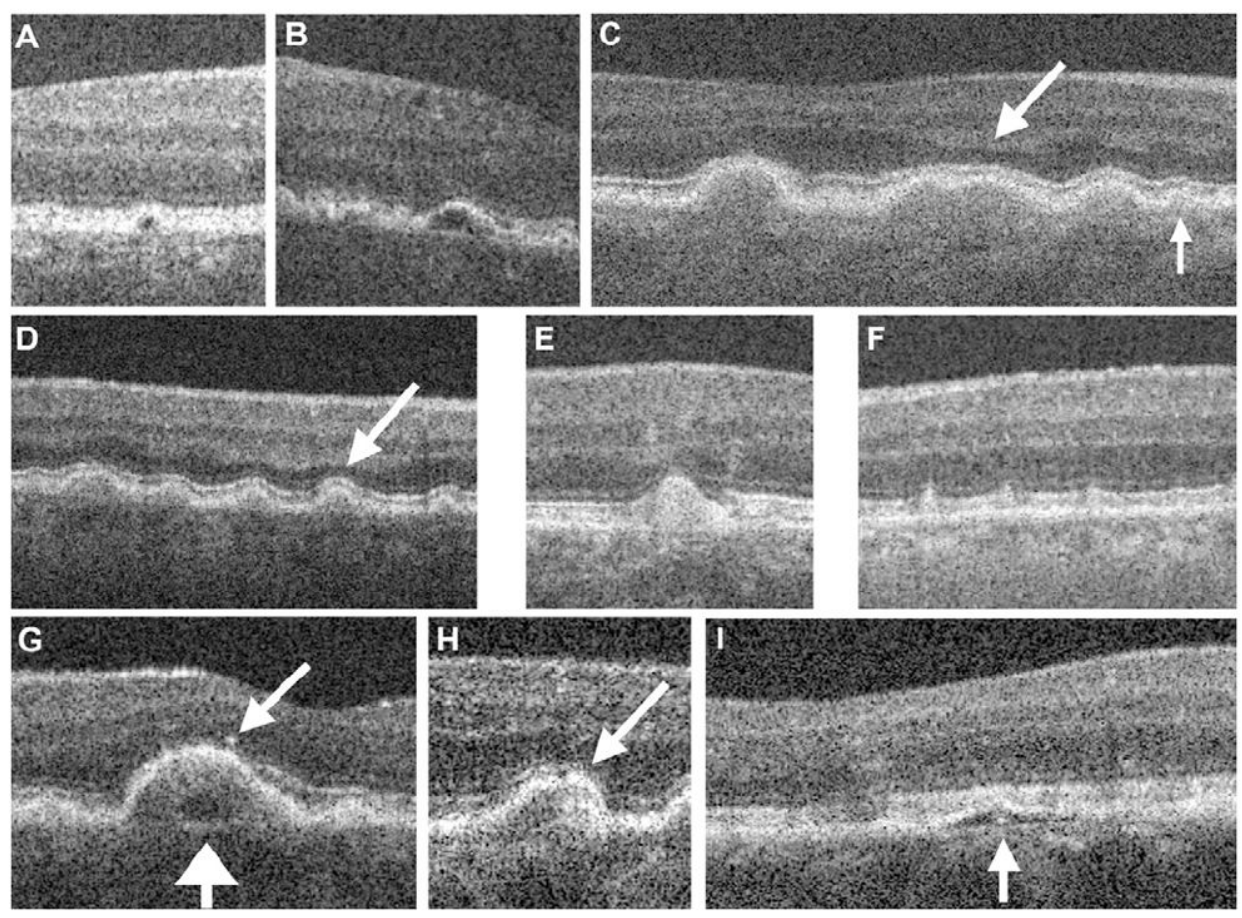

Figure 3.

Spectral-domain optical coherence tomography drusen morphology and retinal morphology related to drusen. The morphology terms are defined in Table 1. To reduce the noise in linear B-scan images, varying numbers of B-scans were summed using Image J software. A, B, Drusen with low reflectivity. C, D, Mid-reflective drusen. Photoreceptor layer thinning overlying the drusen is indicated by the arrows. E, Highly reflective druse. F, Stage 2 subretinal reticular drusenoid deposits are characterized as drusen. G-I. Drusen with cores. G, The low reflective core (wide arrow) is within a mid-reflective drusen with overlying hyperreflective focus (narrow arrow). H, Hyperreflective focus within a mid-reflective druse. I, Low-reflective druse with a core. 


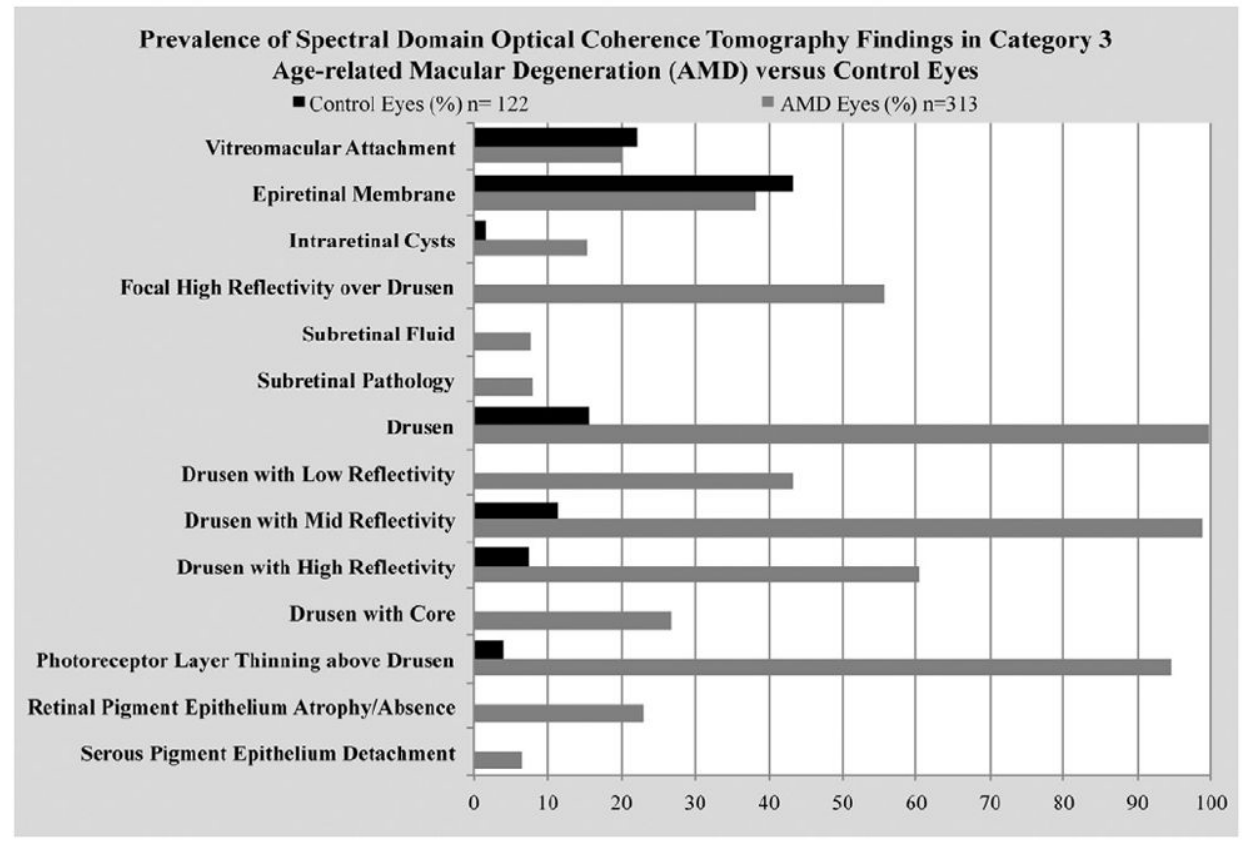

Figure 4.

Prevalence of spectral-domain optical coherence tomography retinal pathologies and drusen characteristics among the 313 category 3 age-related macular degeneration (AMD) study eyes. 


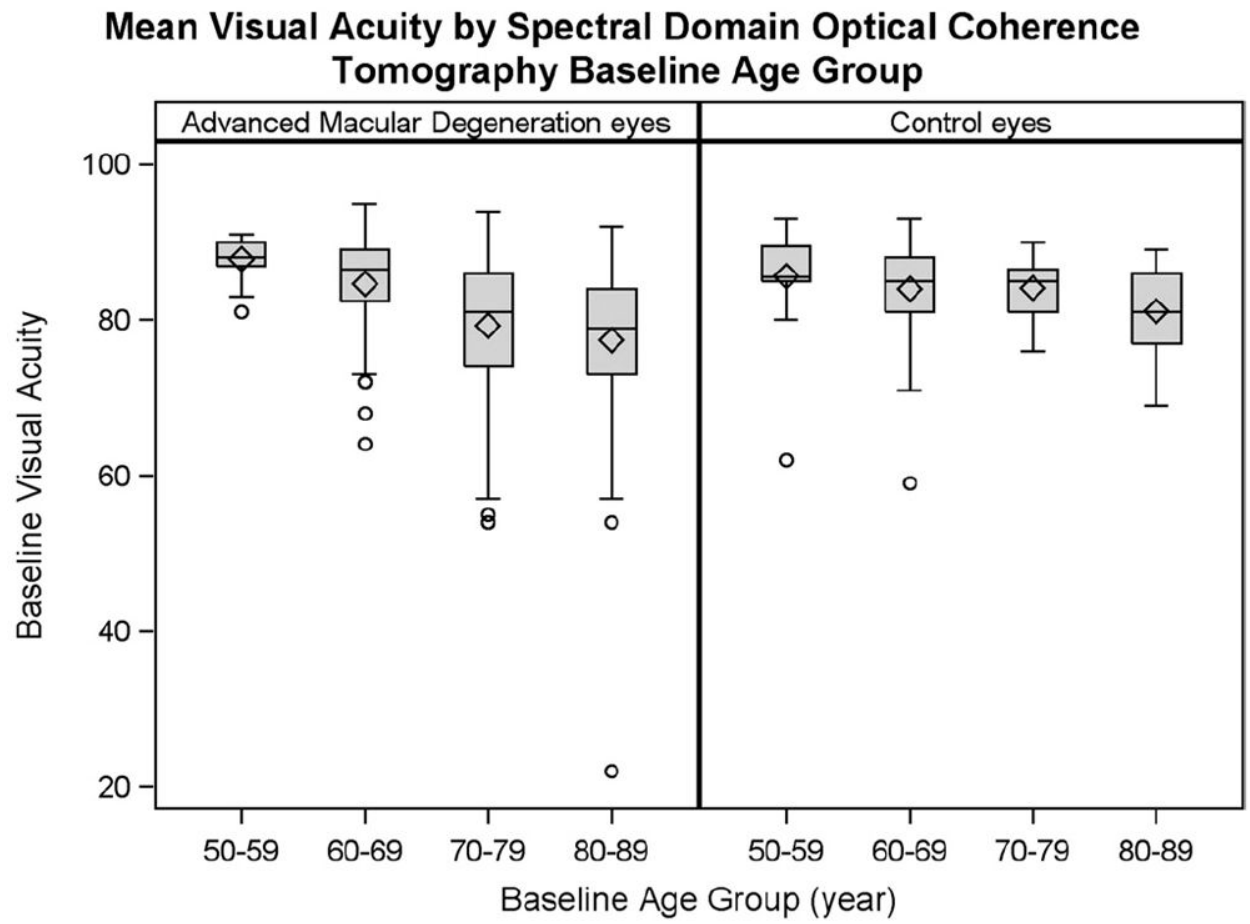

Figure 5.

Effect of age on visual acuity among 313 category 3 age-related macular degeneration (AMD) study eyes and 122 control eyes. The box outlines the interquartile range (25th-75th percentiles), and whiskers extend to the most extreme data point within 1.5 times the length of interquartile range. Values beyond 1.5 times the interquartile range were considered outliers (circles). The mean Early Treatment Diabetic Retinopathy Study value is shown as a diamond within each box, and a horizontal line represents the median. 


\section{Mean Visual Acuity by Foveal Pathology on Spectral Domain Optical Coherence Tomography Scans for Advanced Macular Degeneration Eyes}

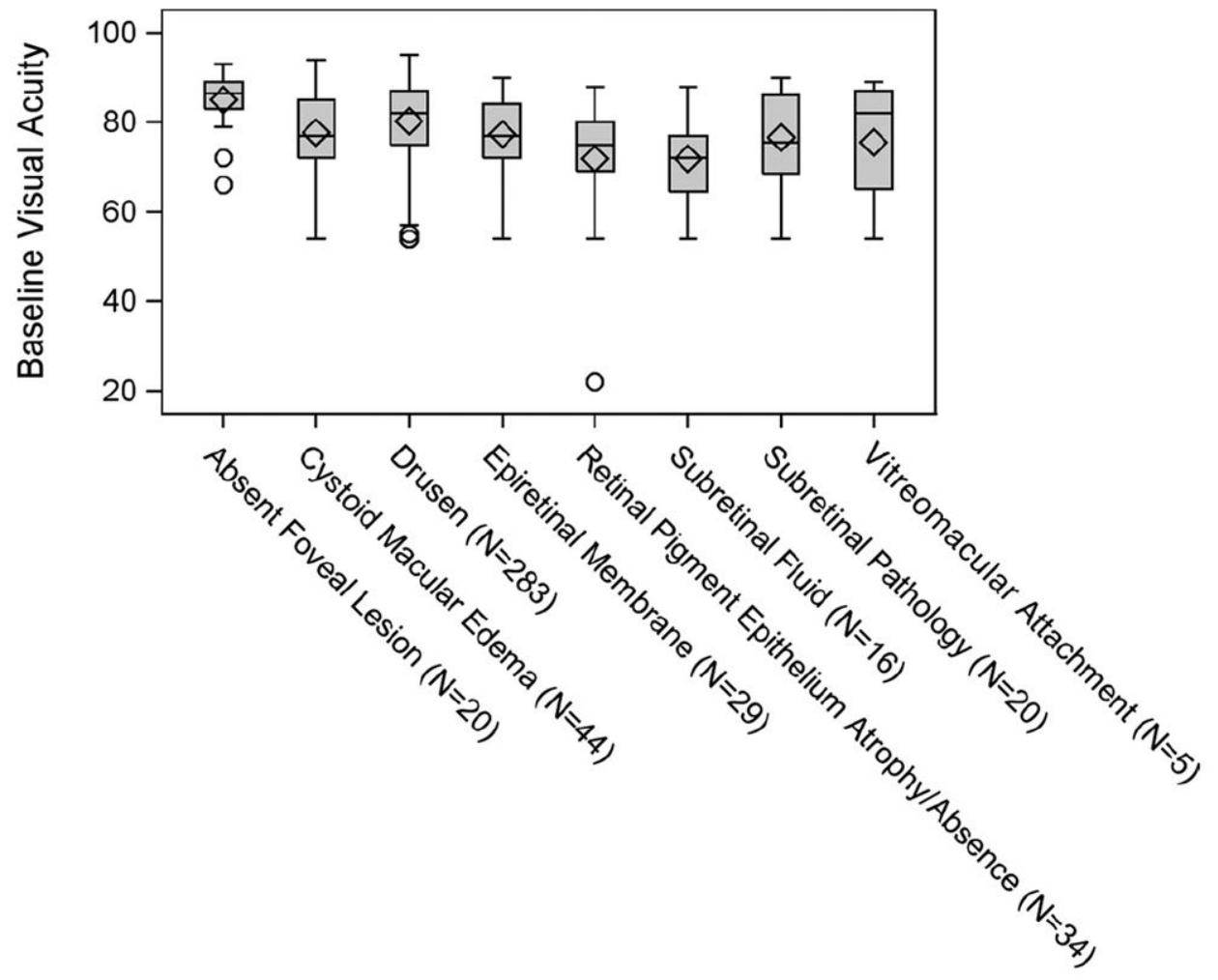

Baseline Foveal Pathology

Figure 6.

Effect of foveal pathologies on visual acuity among 313 category 3 age-related macular degeneration (AMD) study eyes. The mean visual acuity score (adjusted for age) was higher among eyes without foveal pathology than among eyes with foveal pathologies. This difference was significant $(P<0.02)$ in all subgroups except eyes with foveal vitreomacular attachment (VMA; $P=0.1278$ ). Among eyes containing foveal pathology, the subgroup with the highest Early Treatment of Diabetic Retinopathy Study score was eyes with foveal drusen, although the Early Treatment Diabetic Retinopathy Study score was still significantly decreased compared with controls $(P=0.009)$. Eyes containing foveal subretinal fluid and foveal retinal pigment epithelium atrophy or absence had the poorest visual acuities, significantly lower than eyes without foveal pathology $(P<0.0001$ and $P=$ 0.0002 , respectively). When compared with no foveal lesion, there was no difference for VMA. In light of multiple comparisons, there is likely no difference for epiretinal membrane and drusen, but there was a difference in the others: intraretinal cysts, $P=0.002$; subretinal fluid, $P \unlhd 0.0001$; other subretinal lesion, $P=0.002$; and atrophy, $P=0.0002$. The box outlines the interquartile range (25th-75th percentiles), and whiskers extend to the most extreme data point within 1.5 times the length of interquartile range. Values beyond 1.5 times the interquartile range were considered outliers (circles). Diamonds represent the mean Early Treatment of Diabetic Retinopathy Study value (adjusted for age), and the horizontal line within the box indicates the median. Additionally, analysis of variance assumptions of normality and equal variances were violated among eyes with foveal vitreomacular attachment, subretinal fluid, drusen, and retinal pigment epithelium atrophy or 
absence. The analysis of variance model is robust if the sample size is large (as in the case of eyes with foveal drusen), but results may be biased among subgroups with a small number of eyes (e.g., vitreomacular attachment). 